\title{
Exposure to antimicrobials through the milk diet or systemic therapy is associated with a transient increase in antimicrobial resistance in fecal Escherichia coli of dairy calves
}

\author{
C. A. Foutz, ${ }^{* 1}$ S. M. Godden, ${ }^{*}$ J. B. Bender, ${ }^{*}$ F. Diez-Gonzalez, $†$ M. Akhtar, $\ddagger$ and A. Vatulin* \\ *Department of Veterinary Population Medicine, College of Veterinary Medicine, University of Minnesota, Saint Paul 55108 \\ †Department of Food Science and Technology, College of Agricultural and Environmental Sciences, Center for Food Safety, University of Georgia, \\ Griffin 30223 \\ ‡Department of Food Science and Nutrition, University of Minnesota, Saint Paul 55108
}

\begin{abstract}
The objective of this prospective cohort study was to describe the relationship between exposure to antimicrobials, through both the milk diet and systemic therapy, and to describe antimicrobial resistance of fecal Escherichia coli in dairy calves pre- and postweaning. A convenience sample of 15 Minnesota dairy farms was chosen, representing 3 equal cohorts of milk diet fed to preweaned calves: medicated milk replacer (MMR), nonmedicated milk replacer (NMR), or pasteurized nonsaleable milk (PNM). Five newborn calves were enrolled on each farm, with fecal samples collected from each calf at 1, 3, 5, and 16 wk of age. After isolation, 3 colonies of $E$. coli were randomly selected from each sample to determine antimicrobial susceptibility by minimum inhibitory concentration (Sensititer, Thermo Scientific, Waltham, MA) to 8 antimicrobials in 8 classes. The isolate was given an antimicrobial resistance score (ARS) according to the number of antimicrobial classes to which it was resistant. Any isolate resistant to 3 or more antimicrobials was defined as being multidrug resistant (MDR). Relationships between ARS and MDR (dependent variables) and possible explanatory variables were analyzed using mixed multivariable linear and logistic regression models, respectively, with critical $P$-values adjusted for multiple contrasts. Seventy percent of isolates were resistant to sulfadimethoxine. For wk 1 and 3 , the mean ARS values were greatest for fecal $E$. coli from calves fed MMR or PNM compared with NMR, with no difference in ARS values between the MMR and PNM groups at either time point. At wk 5, the mean ARS value was greatest for fecal $E$. coli from calves fed MMR (3.56 \pm 0.45 ; mean $\pm \mathrm{SE})$, intermediate for calves fed PNM $(2.64 \pm 0.45)$, and lowest for calves fed NMR $(1.54 \pm 0.45)$. However, by wk 16 , the
\end{abstract}

Received February 15, 2018.

Accepted June 30, 2018.

${ }^{1}$ Corresponding author: foutz001@umn.edu mean ARS values were $\leq 1.0$ and did not differ among milk diets. Evaluation of the proportion of isolates with MDR mirrored the results of the ARS analysis (MDR more prevalent in MMR and PNM groups preweaning; no difference among milk diets at $16 \mathrm{wk}$ ). There was a tendency for an increase in ARS at wk $5(1.28 \pm 0.70)$, and the odds for MDR in fecal E. coli were estimated to be $5.2(95 \%$ confidence interval $=0.67,35.7)$ and $101.1(95 \%$ confidence interval $=1.15,>999.9)$ higher at wk 3 and 5 if the calf was treated with a systemic antimicrobial within the 14-d period before sampling. These findings suggest that exposure to antimicrobials through the milk diet or systemic therapy may result in a transient increase in resistance in fecal E. coli, but once the antimicrobial pressure is removed, susceptible E. coli are able to flourish again, resulting in an overall decrease in resistance.

Key words: calf, antimicrobial resistance, therapy, milk

\section{INTRODUCTION}

Antimicrobials are a widely used and necessary resource within food animal production systems and can improve animal health and well-being when used appropriately. However, mounting concerns over the emergence of antimicrobial resistance (AMR), which can be caused by mutation or spread from one bacterium to another by the mobilization of resistance genes on plasmids, can threaten both animal and human health (McEwen and Fedorka-Cray, 2002). Of particular concern are medically important antimicrobials that are used in both veterinary and human medicine. As such, food animal agriculture must carefully examine how antimicrobials are used and adopt good stewardship practices designed to reduce the risk of developing newly resistant bacteria that could be transmitted from animals to humans.

In preweaned dairy calves, 2 common potential sources of antimicrobial exposure include systemic 
therapy for the treatment of infectious disease and the milk diet. According to the USDA's National Animal Health Monitoring System (NAHMS) 2007 Dairy study (USDA, 2010), 31.4 and $8.5 \%$ of preweaned and weaned heifers, respectively, were treated with a systemic antimicrobial, with the more commonly used antimicrobial agents including tetracyclines, tylosin, tilmicosin, florfenicol, cephalosporins, fluoroquinolones, and penicillins. As for the question of potential exposure of preweaned heifers to antimicrobials through the milk diet, the USDA (2014) dairy study reported that $37.6 \%$ of dairy operations fed medicated milk replacer, with $49.9 \%$ of operations feeding any milk replacer. Medicated milk replacer is milk replacer that contains various types of approved medications for treatment of disease, as regulated under the US Food and Drug Administration (FDA) Veterinary Feed Directive (FDA, 2015). Of the $37.6 \%$ of dairies feeding medicated milk replacer in the 2014 USDA Dairy study, 1.6, 4.9, and $9.0 \%$ of operations fed products containing chlortetracycline, oxytetracycline, or neomycin and oxytetracycline, respectively. Other reported medications included decoquinate, lasalocid, and monensin. The same study reported that unpasteurized or pasteurized (saleable or nonsaleable) milk was fed on 55.7 and $7.4 \%$ of dairy operations, respectively. Nonsaleable milk may include milk from just-fresh cows or milk harvested from hospital cows being treated with antimicrobials for any reason (e.g., mastitis) that must be discarded because of required milk withholding times due to the presence of antimicrobial residues. On-farm pasteurization of nonsaleable milk is important to reduce exposure to infectious bacteria that may be in milk but it does not inactivate antimicrobials that may be in the milk (Aust et al., 2013).

One important question surrounding the management of dairy calves is whether exposure of calves to antimicrobials, either through feed or systemic therapy, will increase the shedding of resistant pathogens in feces or cause the development of newly resistant enteric pathogens. A limited number of studies have described the association between systemic antimicrobial therapy and AMR in enteric pathogens in preweaned calves. In one cross-sectional study of preweaned calves from 8 commercial New York dairy farms, Pereira et al. (2014a) reported increased AMR in fecal E. coli in calves previously treated with systemic antimicrobials for clinical disease compared with untreated calves. For example, treatment of a calf with enrofloxacin was significantly associated with a higher number of nonsusceptible fecal E. coli. Among calves treated with ceftiofur, $97 \%$ were resistant to 3 or more antimicrobials versus $73 \%$ for calves not treated with ceftiofur. However, an important study limitation was that each calf was sampled only once following treatment, with the median numbers of days from treatment with ceftiofur and enrofloxacin to rectal swab sampling of calves being $16 \mathrm{~d}$ (range: 1-39) and $12 \mathrm{~d}$ (range: 6-44), respectively. As such, the persistence of changes in resistance patterns in treated (vs. untreated) calves over longer periods could not be described.

Some studies have investigated changes in antimicrobial resistance in the intestinal bacteria of preweaned calves being fed milk (Langford et al., 2003; Pereira et al., 2014b) or milk replacer (Thames et al., 2012) when increasing levels of antimicrobials were experimentally added to the milk diet. Langford et al. (2003) and Pereira et al. (2014b) both reported increased phenotypic resistance in intestinal bacteria for calves on medicated milk diets, whereas Thames et al. (2012) did not observe an effect of diet on genes encoding for antimicrobial resistance in calf feces. However, limitations of these studies included the use of a relatively small number of calves under conditions of artificial exposure to antimicrobials. Furthermore, Langford et al. (2003) and Pereira et al. (2014b) only sampled calves for a short period ( $\leq 6$ wk of age) while on the milk diet. Conversely, Thames et al. (2012) sampled calves both pre- and postweaning (wk 6, 7, and 12).

Clinical trials performed using naturally adulterated milk diets (Aust et al., 2013) have reported that a greater proportion of resistant fecal $E$. coli isolates were recovered from calves fed raw or pasteurized nonsaleable waste milk than from calves fed raw or pasteurized bulk tank milk. However, that study did not investigate the effect of systemic antimicrobial therapy (treated calves were excluded from the analysis) and did not follow calves beyond $56 \mathrm{~d}$ of age. More recently, in a controlled trial of 52 calves on one research facility in Minnesota, Maynou et al. (2017b) reported that feeding pasteurized nonsaleable milk to calves increased the presence of phenotypic resistance to ampicillin, cephalothin, ceftiofur, and florfenicol in fecal E. coli compared with calves fed nonmedicated MR. However, the presence of resistance to sulfonamides, tetracyclines, and aminoglycosides was common in dairy calves independent of their milk-feeding source, suggesting that factors other than the feeding source are involved in the emergence of antimicrobial resistance. Limitations of this study included the fact that it excluded any calf treated with systemic antimicrobials (52\% of 52 study calves were excluded), resulting in a limited sample size. Furthermore, it only sampled calves to $56 \mathrm{~d}$ of age.

Several observational studies have been completed under field conditions. In one cross-sectional study using cohorts of 30 calves each from 6 commercial farms, 
Berge et al. (2005) reported a strong association between antimicrobial resistance in fecal E. coli and the usage of medicated milk replacer, as well as an association with calf age and systemic antimicrobial usage in preweaned calves. Strengths of this study included that it did represent conditions of natural exposure and it examined both systemic and dietary sources of exposure to antimicrobials. However, limitations included the fact that only medicated milk replacer and nonsaleable (hospital) milk diets were studied, and calves were not followed beyond weaning. In another a recent observational study of calves from 8 commercial dairy farms in Spain, Maynou et al. (2017a) reported that a greater number of fecal $E$. coli were resistant to enrofloxacin, florfenicol, and streptomycin, and more multidrug-resistant $E$. coli phenotypes were isolated in feces of calves from 4 farms that fed waste milk versus calves from 4 farms that fed nonmedicated milk replacer, when samples were collected at 6 wk of age. However, when study animals were resampled at $1 \mathrm{yr}$ of age, there was no difference in resistance patterns between the 2 milk cohorts and, in general, resistance had decreased in both groups. Strengths of the latter study included evaluating resistance in fecal pathogens of calves under conditions of natural exposure, as well as over a long period beyond weaning. However, one limitation was that it did not investigate or control for the potential effects of systemic antimicrobial therapy during the period of observation.

Most of the aforementioned studies demonstrate that fecal pathogens shed by preweaned calves show increased antimicrobial resistance when exposed to antimicrobials from the milk diet or systemic antimicrobial treatments. However, many of these studies were not conducted under conditions of natural exposure, many excluded consideration of systemic antimicrobial therapy, and most did not study calves after weaning, beyond the milk-feeding phase. This latter point may be an important consideration, because these studies were not able to determine whether increases in resistance observed during the preweaning period represented transient or long-lasting effects. Singer et al. (2008) assessed the effects of systemic treatment of adult cows with ceftiofur on antimicrobial resistance patterns of fecal E. coli. Those researchers reported that before treatment, fecal $E$. coli was susceptible to ceftiofur; however, immediately following treatment and during the treatment protocol, there were decreased bacterial counts (decrease in the susceptible population) and more resistance. However, by d 7 after the treatment was concluded, the susceptible $E$. coli began to flourish again, leading to a decrease in the overall resistance patterns of E. coli cultured from fecal samples (Singer et al., 2008). As such, systemic treatment with ceftiofur in adult dairy cattle caused only a transient effect. It is possible that the same transient effects could hold true with respect to calves exposed to antimicrobials either through systemic therapy or the milk diet.

The objective of this prospective cohort study was to describe, under conditions of natural exposure, the relationship between exposure to antimicrobials, both through the milk diet and through systemic therapy, and antimicrobial resistance patterns of fecal E. coli in dairy calves pre- and postweaning. We hypothesized that antimicrobial resistance in fecal $E$. coli would be highest early in life for preweaned calves exposed to antimicrobials through the milk diet, or via recent systemic therapy with antimicrobials, but that this antimicrobial resistance would be transient, decreasing after weaning when the antimicrobial exposure, through either the milk diet or systemic antimicrobial therapy, was removed or reduced, respectively.

\section{MATERIALS AND METHODS}

\section{Farm Enrollment}

A convenience sample of 15 commercial Holstein dairy farms in Minnesota were recruited for this cohort study, being purposefully selected based on their willingness to participate with study activities, and to achieve 5 herds representing each of the following 3 milk diet cohorts: (1) pasteurized nonsaleable milk (PNM, $\mathrm{n}=5$ herds), (2) medicated milk replacer (MMR, $\mathrm{n}=$ 5 herds), and (3) nonmedicated milk replacer (NMR, $\mathrm{n}=5$ herds; control group).

\section{Enrollment and Fecal Sampling of Calves}

On-farm activities for this project were approved by the University of Minnesota Institutional Animal Care and Use Committee. Each farm was visited weekly between June and October 2009. Upon initiating the study, the first 5 liveborn heifer calves born on each farm were enrolled. Heifer calves were enrolled within the first week of life with the goal of enrolling 5 calves for each farm. On the day of enrollment, fecal samples (10 g) were collected directly from the rectum by a study technician wearing new disposable gloves. Fecal samples were placed immediately into a vial containing a buffer solution (Para-Pak C\&S, Meridian Bioscience Inc., Cincinnati, $\mathrm{OH}$ ), and the vial was then placed into a cooler on ice, and transported to the College of Veterinary Medicine, University of Minnesota (St. Paul, $\mathrm{MN}$ ) where the samples were refrigerated overnight at $4^{\circ} \mathrm{C}$. Fecal samples were collected from each calf at 
4 time points, including wk 1 (enrollment), wk 3, wk 5 (before weaning), and wk 16 (after weaning). Farm staff recorded birth dates, weaning dates, death or culling dates (if applicable), and treatment dates, as well as all types of medical treatments administered to study calves between enrollment and 16 wk of age. Study technicians collected feed tags from all milk replacer, starter pellet, and grower pellet formulations being fed on each farm. Producers within the PNM cohort were asked to collect and freeze $\left(-20^{\circ} \mathrm{C}\right)$ milk samples approximately every 2 wk throughout the 16-wk study period to allow for antimicrobial residue testing.

\section{E. coli Isolation from Fecal Samples}

Fresh fecal samples were serially diluted using PBS. The fourth and fifth dilutions were plated in duplicate on Petrifilm E.coli/Coliform Count Plates (3M, Saint Paul, MN) to select for Escherichia coli and incubated at $37^{\circ} \mathrm{C}$ for $24 \mathrm{~h}$. After $24 \mathrm{~h}$, the plates were inspected for presumptive E. coli colonies. If no colonies were identified, the Petrifilm plate was incubated for an additional $24 \mathrm{~h}$ and reassessed.

Three E. coli colonies from each Petrifilm plate were selected and streaked onto sorbitol MacConkey agar plates (Neogen Inc., Lansing, MI) using standard aseptic technique and incubated at $37^{\circ} \mathrm{C}$ for $24 \mathrm{~h}$. One isolated colony from each plate was used to inoculate a tube of tryptic soy broth (TSB; Neogen Inc.). The TSB culture was incubated at $37^{\circ} \mathrm{C}$ overnight. After incubation, $500 \mu \mathrm{L}$ of the TSB culture was pipetted into 500 $\mu \mathrm{L}$ of glycerol to make a stock culture in duplicate. The stock cultures were then frozen and stored at $-80^{\circ} \mathrm{C}$.

\section{Antimicrobial Resistance Testing and Confirmation of $E$. coli Isolates}

Samples were aseptically streaked onto sheep blood agar plates and incubated at $37^{\circ} \mathrm{C}$ overnight. Using a sterile cotton swab, a few colonies were removed from the sheep blood agar plates and inoculated into a tube of TSB broth. This tube was then placed into a calibrated Sensititer SWIN Software System (Thermo Scientific, Waltham, MA) to ensure a turbidity of $\sim 10^{8}$. If the turbidity was low, a few more colonies were added to the TSB tube using the same technique and the tube was rechecked until the proper turbidity was achieved. If the turbidity was too high, a small amount of sterile saline was added aseptically, or the process was redone with a new tube. Using a sterile $10-\mu \mathrm{L}$ inoculation loop, one loop full of the TSB culture was removed and inoculated into a sterile tube of Mueller Hinton broth (Neogen
Inc.), which was used for autoinoculation of the 96-well BOPO6F Sensititer MIC plate (Thermo Scientific). These inoculated plates were covered and incubated at $37^{\circ} \mathrm{C}$ for 18 to $24 \mathrm{~h}$, at which time they were read. The plates were manually assessed for bacterial growth within each well using a Sensititer plate reader. The panel included 18 antimicrobials: ampicillin, ceftiofur, chlortetracycline, clindamycin, danofloxacin, enrofloxacin, florfenicol, gentamicin, neomycin, oxytetracycline, penicillin, spectinomycin, sulfadimethoxine, tiamulin, tilmicosin, trimethoprim/sulfamethoxazole, tulathromycin, and tylosin. The recorded MIC values and the interpretation of the values were reported as sensitive $(\mathbf{S})$, intermediate $(\mathbf{I})$, or resistant $(\mathbf{R})$. The breakpoints for resistance were interpreted according to the Clinical and Laboratory Standards Institute (CLSI, 2008, 2015, 2018) and are reported in Table 1. The MIC cut-offs were chosen by antimicrobial agent followed by species and bacteria (either E. coli or Enterobacteriaceae). For florfenicol, there were no available cut-offs for $E$. coli or Enterobacteriaceae, so the cut-off for bovine respiratory pathogens was used. Confirmatory tests (e.g., Simmons citrate agar slant test and a urea agar slant test) were conducted to verify that the isolates were $E$. coli. The 2 types of agar were inoculated and incubated at $37^{\circ} \mathrm{C}$ for $24 \mathrm{~h}$. At this point, they were assessed for changes consistent or not with E. coli. A positive Simmons citrate agar slant test is indicated by a change in agar color from orange to bright pink, and a positive urea agar slant test is indicated by a change in agar color from dark green to blue. An isolate would be consistent with $E$. coli when found to be negative for both of these tests.

\section{Antimicrobial Residue Testing of Nonsaleable Milk Samples}

Although we attempted to collect milk samples from all 5 PNM farms, milk samples were available for testing from only 3 of the 5 PNM study farms. Three samples from each of the 3 PNM farms collecting nonsaleable milk samples were sent to DQCI Services (Mounds View, MN) for antimicrobial residue testing. The Charm II (tetracyclines) and Charm SL ( $\beta$-lactams) tests were run on all of the samples according to the manufacturer's guidelines (Charm Sciences Inc., Lawrence, MA) using positive and negative controls for quality assurance purposes. Additionally, HPLC was performed on a selection of the collected samples, according to a standard HPLC protocol with a known control/reference peak allowing for comparison with other identified peaks. 


\section{Data Analysis}

All analysis was performed using SAS software (version 9.4; SAS Institute Inc., Cary, NC). The database structure was as follows: Herd $(\mathrm{n}=15)$, calf $(\mathrm{n}=74$, with 5 calves per each herd), sampling time (wk 1, 3, 5, and 16 for each calf), and isolate ( 3 E. coli isolates per calf within each sampling time). The main dependent variable used to describe antimicrobial resistance was the antimicrobial resistance score (ARS). A secondary dependent variable of interest was whether an isolate was multidrug resistant (MDR; yes/no). An ARS value was calculated for each $E$. coli isolate by the following process: Eight of the 18 antimicrobials on the MIC panel were selected for evaluation, representing 8 different classes of antimicrobials: ampicillin (class: penicillin), ceftiofur (cephalosporin), enrofloxacin (floroquinolone), florfenicol (phenicol), gentamicin (aminoglycoside), oxytetracycline (tetracycline), sulfadimethoxine (sulfonamide), and trimethoprim/sulfamethoxazole (folate pathway inhibitor). These antimicrobials were chosen to include the commonly used antimicrobial classes of clinical dairy medicine (specifically dairy heifer calves) along with antimicrobials that were specifically used as treatments during the sampling period or in the medicated milk replacers given to the calves. Using these 8 selected antimicrobials, an antibiogram was formulated, this being a string of letters (S, I, R) that indicated the resistance pattern of a particular isolate to each of the 8 antimicrobials. For the purposes of our analysis, "I" readings were categorized as $\mathrm{S}$. The total number of " $R$ " patterns was then summed to give the final ARS value for that isolate (possible ARS value $=0$ to 8 ). Any isolate resistant to 3 or more antimicrobials/antimicrobial classes was defined as being MDR. This cutoff was chosen by assessment of a natural break in the data along with what is seen in the current literature (Magiorakos et al., 2012).

Descriptive statistics were generated to describe study herd characteristics, the number of calves enrolled, the mean age (d) at each fecal sampling event (wk 1, 3, 5, 16), the proportion of calves treated systemically with an antimicrobial before a given fecal sampling event, and the mean days, before fecal sampling, that systemic antimicrobial treatments were administered. Descriptive data were also generated to describe the proportion of $E$. coli isolates that were susceptible, intermediate, or resistant, for each of the 8 antimicrobials considered. Finally, descriptive statistics were generated to describe the milk diet cohort, date of collection, the average ARS value for $E$. coli isolates, and the proportion of isolates that were MDR. The ARS variable was determined to be normally distributed by examination of skewness statistic, as well as by plotting a frequency histogram.

Univariable linear regression (using the MIXED procedure) was initially used to describe the relationship between the ARS (continuous dependent variable) and each of the following possible explanatory variables of interest: (1) milk diet (MMR, NMR, PNM), (2) sampling time (wk 1, 3, 5 or 16), (3) calf housing (individual vs. group), (4) lactating cow housing (freestall vs. tiestall), (5) milking herd size (number of milking cows), (6) herd average milk production ( $\mathrm{kg} / \mathrm{cow}$ per day), and (7) whether the calf was treated with a

Table 1. Antimicrobial classes and agents evaluated along with the utilized breakpoints and sources

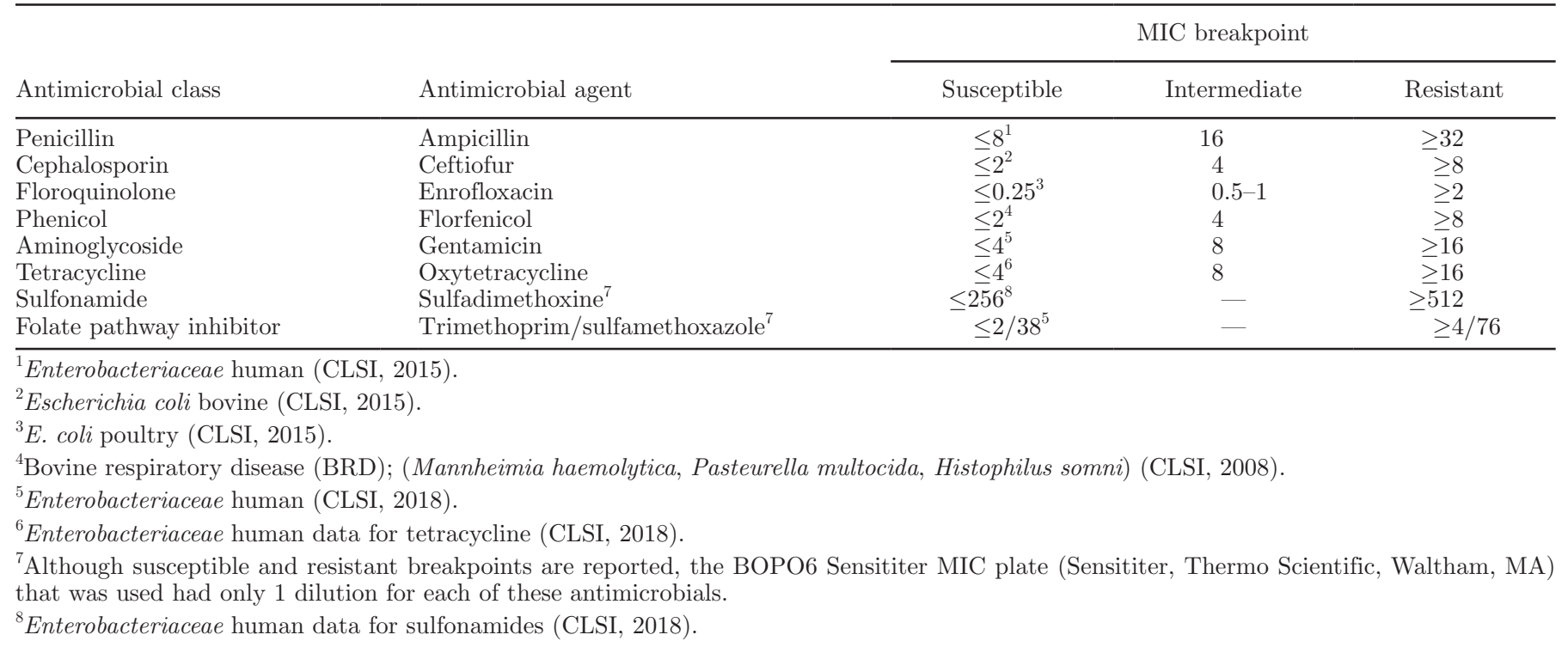


systemic antimicrobial within the 14-d period preceding the fecal sampling event (yes/no). In selecting the latter parameter, we first examined this parameter in several different ways, including as both a continuous and categorical variable, and determined that both were significantly associated with the AMR outcomes. However, because the interval (days) between systemic therapy and fecal sampling had a strong bimodal distribution, the decision was made to categorize the results. In selecting the cutpoint by which to categorize this parameter, we created a variety of different categorical variables investigating the nature and strength of association between ARS and whether treatment with a systemic antimicrobial occurred during the following periods preceding sample collection: $3,5,7,10,14,20$, 30,42 , or $120 \mathrm{~d}$ preceding the fecal sampling event, or never. After comparing the models generated using these different treatment-to-sampling intervals, it was determined that a treatment-to-sampling interval of $\leq 14 \mathrm{~d}$ was most strongly associated with ARS, yielding the best model fit (smallest -2 Log-likelihood statistic value).

Any explanatory variable significant at $P<0.20$ in the univariable analysis was carried forward to offer into the final multivariable mixed linear model, and then subjected to a stepwise backward elimination process whereby final significance was declared at $P<$ 0.05. The mixed model also included 3 random effects: herd, calf, and sampling time(calf). The presence of all possible 2-way interactions was then explored. If an interaction was detected, then the data were reanalyzed after stratification. Models were compared during the model building process using the -2 Log-likelihood statistic. We evaluated the fit of the final mixed linear model $(\mathrm{ARS}=$ dependent variable $)$ by examination of residual plots as well as quantile-quantile (Q-Q) plots. The residual plots showed the data points aligned almost perfectly along the diagonal line of fit. A frequency histogram of the residuals for ARS and QQ plots showed equal variance around the mean value of zero. This process was completed for both the unconditional (marginal) and the conditional residuals, where the conditional residuals also consider the random effects. The various plots described above were generated using the "plots = all" option within the Proc MIXED statement in SAS.

In a separate but similar backward stepwise approach, a multivariable mixed logistic regression model (using the GLIMMIX procedure) was developed to describe the relationship between odds for an isolate being MDR (yes/no; dependent variable) and each of the aforementioned possible explanatory variables of interest; milk diet (MMR, PNM, NMR), sampling time (wk 1, 3, 5, 16), previous systemic antimicrobial treatment within $14 \mathrm{~d}$ of sampling (yes/no), calf housing (group vs. individual), lactating cow housing (freestall vs. tiestall), herd average milk production ( $\mathrm{kg} / \mathrm{cow}$ per day), and herd size (number of lactating cows). The final mixed logistic model included 3 random effects: herd, calf, and sampling time(calf).

\section{RESULTS}

\section{Study Herd Characteristics}

All 15 study herds were closed systems (i.e., they did not introduce new animals from other sources). Median herd size was 265 lactating cows (range: 52 to 1,277 ) with an average milk yield of 34.0 (SD 8.3) kg per cow per day. Mean milking herd size varied by milk cohort type, being smaller for herds feeding NMR (68 cows) than for those feeding MMR (302 cows) or PNM (919 cows). For the NMR herds, 3 herds used tiestall housing and 2 herds used freestall housing for adult cows. All herds in the MMR and PNM groups used freestall housing for adult cows. Fourteen farms raised preweaned calves in individual hutches or individual pens within a barn, delivering milk by either nipple bottle or bucket. One farm feeding MMR raised the preweaned calves in a group pen with an automated feeder. Following weaning, calves on all farms were housed in group pens. Antimicrobials fed in the commercial milk replacer formulations for the MMR herds included neoterramycin, neomycin, oxytetracycline, and chlortetracycline. Antimicrobial residues detected via HPLC in nonsaleable milk samples collected from 3 of the 5 PNM herds included ampicillin, cephapirin, penicillin, ceftiofur, and ceftiofur metabolites. Because of the identification of multiple antimicrobial residues in each sample, fractions, but not specific concentrations, were reported. The Charm II test, which screens for tetracyclines, yielded "none found" (no tetracyclines present to the limit of detection) in any of the submitted samples. The Charm SL test, which screens for $\beta$-lactams, yielded 4 positive samples out of 9 samples submitted (3 from each farm). No antimicrobials were reported to be fed in the calf starter pellet (fed preweaning); however, chlortetracycline was reported to be fed in the calf grower (fed postweaning) for one of the PNM herds.

\section{Study Calf Characteristics}

Seventy-four calves were enrolled in the study; on one of the smaller farms (NMR), only 4 calves were enrolled because of study timeline constraints. On one of the 
larger farms (MMR), 2 calves died early in the study, at 17 and $20 \mathrm{~d}$ of age, respectively, before the wk 5 and wk 16 samples could be collected. These calves were replaced by enrolling 2 new calves of similar age to allow for a complete set of 5 calves from this herd. Three other enrolled calves were lost from the study at ages $84 \mathrm{~d}$ (died; MMR), $85 \mathrm{~d}$ (died; MMR), and sometime between 35 and $70 \mathrm{~d}$ (sold; NMR), respectively. However, because most of their records were complete (with the exception of the wk 16 sample event) and because there was insufficient time to replace these older calves, we included their isolate results from wk 1, 3 and 5 in the final analysis.

For all calves, the mean $( \pm \mathrm{SD})$ age at the wk 1, 3, 5 and 16 fecal sampling events was $4.6( \pm 2.0), 18.6$ $( \pm 2.0), 32.6( \pm 2.0)$, and $107.8( \pm 6.1) \mathrm{d}$ of age, respectively (Table 2). All calves were weaned between the wk 5 (35 d) and wk 16 (112 d) sampling event, with an average age at weaning of $52.6 \mathrm{~d}(\mathrm{SD} \pm 9.4$; range: 35 to 60 ). There was an average interval of approximately $59 \mathrm{~d}$ between weaning and the final wk 16 postweaning sampling event. Twenty-two of the 74 calves (29.7\%) received one or more systemic antimicrobial treatments for either clinical illness or prophylaxis during the 16wk study period, with $40 \%(10 / 25), 28 \%(7 / 25)$, and $20.8 \%(5 / 24)$ calves receiving systemic antimicrobial treatment in the MMR, PNM, and NMR groups, respectively. Antimicrobials reported to be used for systemic therapy included penicillin, ampicillin, ceftiofur, enrofloxacin, and florfenicol. Of the 22 systemically treated calves, a breakdown of antimicrobials used for systemic therapy was as follows: MMR herds: penicillin $(\mathrm{n}=7)$, ceftiofur $(\mathrm{n}=1)$, florfenicol $(\mathrm{n}=1)$, and enrofloxacin $(\mathrm{n}=2)$; PNM herds: ampicillin $(\mathrm{n}=4)$, florfenicol $(\mathrm{n}=2)$; NMR herds: penicillin $(\mathrm{n}=4)$, florfenicol $(\mathrm{n}=1)$. For those calves treated systemically, the average age at first treatment with an antimicrobial was $8.8 \mathrm{~d}(\mathrm{SD} \pm 8.6$; range: 0 to 34$)$. Table 2 describes the timing of systemic antimicrobial treatment events relative to each of the 4 fecal sampling events.

\section{AMR Patterns of Fecal E. coli Isolates}

A total of 293 fecal samples were collected from the 74 study calves, with 3 presumptive $E$. coli isolates selected from each sample, for a total of 879 presumptive E. coli isolates. After confirmatory testing, 871 E. coli isolates were tested from the 74 calves.

Antimicrobial MIC test results for the 871 fecal $E$. coli isolates are presented in Table 3. The majority of isolates were resistant to sulfadimethoxine (70\%) and almost half were resistant to florfenicol (44\%). Resistance to trimethoprim/sulfamethoxazole, ceftiofur, gentamicin, and enrofloxacin was $33,27,18$, and $0.7 \%$, respectively. All isolates were susceptible or had intermediate susceptibility to ampicillin and oxytetracycline. Eighteen different resistance patterns (r-types) were identified, with resistance to 1 to 6 antimicrobials. Thirty-six percent of the isolates were resistant to 3 or more antimicrobials (Figure 1) and so were considered MDR. Of the 294 MMR isolates, 50\% were MDR and the most common r-type was florfenicol-gentamicinsulfadimethoxine-trimethoprim/sulfamethoxazole). Of the 295 PNM isolates, $47 \%$ were MDR and the most common r-type was ceftiofur-florfenicol-sulfadimethoxine. Finally, of the 287 NMR isolates, $10 \%$ were MDR and the most common r-type was ceftiofur-florfenicolsulfadimethoxine. These MDR isolates spanned 11 different r-types. The most common MDR r-type was ceftiofur-florfenicol-sulfadimethoxine-trimethoprim/ sulfamethoxazole), with this r-type accounting for $9 \%$ of the isolates. Of those, $51 \%$ were from MMR isolates, $39 \%$ were from PNM isolates, and $10 \%$ were from NMR isolates. There were 5 varied r-types in the isolates resistant to 3 antimicrobials. All were resistant to sulfadimethoxine, whereas $86 \%$ were resistant to florfenicol. There were 4 varied r-types in the isolates resistant to 4 antimicrobials. Again, all were resistant to sulfadimethoxine, $97 \%$ were resistant to florfenicol, and $86 \%$ were resistant to trimethoprim/sulfamethoxazole. Additionally, $68 \%$ of these r-types were resistant

Table 2. Description of systemic antimicrobial treatment events relative to fecal sampling events

\begin{tabular}{lcccc}
\hline $\begin{array}{l}\text { Fecal sample } \\
\text { event }\end{array}$ & $\begin{array}{c}\text { No. of } \\
\text { calves sampled }\end{array}$ & $\begin{array}{c}\text { Age at } \\
\text { sampling }\end{array}$ & $\begin{array}{c}\text { Cumulative no. (\%) treated with } \\
\text { a systemic antimicrobial before } \\
\text { the fecal sampling event }\end{array}$ & $\begin{array}{c}\text { Interval between the most recent } \\
\text { antimicrobial treatment and the } \\
\text { fecal sampling event }\end{array}$ \\
\hline Week 1 & 74 & $4.6(2.0 ; 1$ to 8$)$ & $11(14.9)$ & $4.2(2.8 ; 1$ to 8$)$ \\
Week 3 & 74 & $18.6(2.0 ; 15$ to 22$)$ & $20(27.0)$ & $11.9(7.2 ; 2$ to 22) \\
Week 5 & 74 & $32.6(2.0 ; 29$ to 36$)$ & $22(29.7)$ & $22.8(10.2 ; 1$ to 36$)$ \\
Week 16 & $71^{2}$ & $107.8(6.1 ; 98$ to 119$)$ & $22(31.0)$ & $88.7(23.4 ; 41$ to 119$)$ \\
\hline
\end{tabular}

\footnotetext{
${ }^{1}$ Values reported are mean (SD; minimum to maximum).

${ }^{2}$ Three calves lost during study (2 died, 1 sold) between the wk 5 and wk 16 sampling.
} 


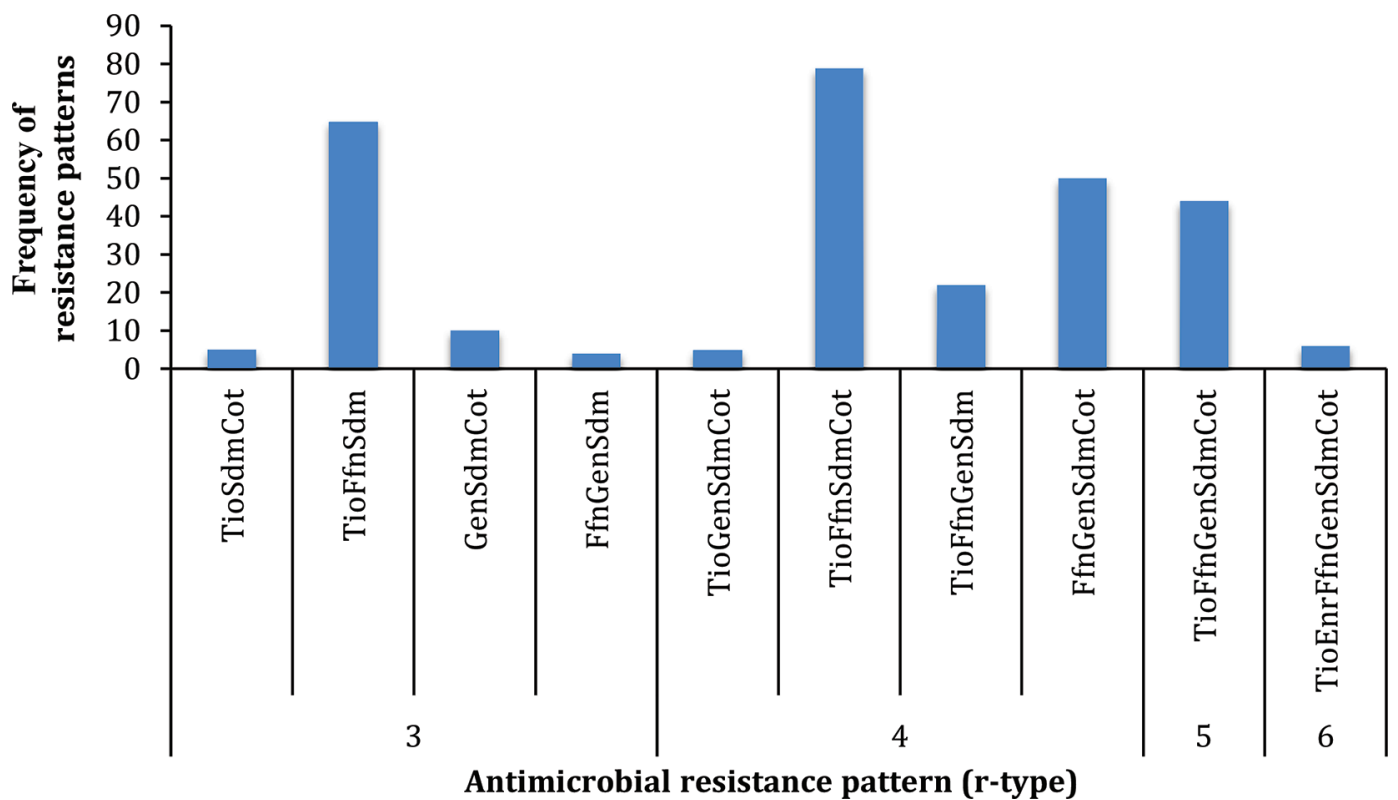

Figure 1. Frequency of antimicrobial resistance patterns (r-type) observed in the multidrug-resistant isolates. Cot $=$ trimethoprim/sulfamethoxazole; Enr = enrofloxacin; Ffn = florfenicol; Gen = gentamicin; Sdm = sulfadimethoxine; Tio = ceftiofur. Color version available online

to ceftiofur and $49 \%$ were resistant to gentamicin. One r-type accounted for all 44 isolates resistant to 5 antimicrobials, including ceftiofur, florfenicol, gentamicin, sulfadimethoxine, and trimethoprim/sulfamethoxazole. Enrofloxacin resistance was only found in the ceftiofurenrofloxacin-florfenicol-gentamicin-sulfadimethoxinetrimethoprim/sulfamethoxazole r-type, which was the only r-type resistant to 6 antimicrobials and was found in only 6 isolates $(0.69 \%)$.

\section{ARS and MDR of Fecal E. coli in Relation to Milk Diet, Systemic Antimicrobial Therapy, and Calf Age}

The unadjusted mean (SD) ARS and the proportion of $E$. coli isolates that were MDR are presented by milk diet and sample time in Table 4. Univariable analyses indicated that the following explanatory variables were associated with both the ARS and MDR dependent variables: milk diet fed, sampling time, systemic antimicrobial treatment in the 14 -d period preceding fecal sample collection, lactating cow housing, and herd size (Table 5). Specifically in the univariable models, ARS and odds for MDR were increased in fecal E. coli from calves in MMR and PNM herds (vs. NMR), in isolates from fecal samples collected at 1, 3, or 5 wk of age (vs. $16 \mathrm{wk}$ ), if the calf had received systemic antimicrobial therapy in the 14-d period preceding fecal sample collection, in herds that housed lactating cows in freestalls (vs. tiestalls) and in larger herds.

In the final multivariable mixed linear regression model predicting ARS as the dependent variable, only 3 explanatory variables of interest - milk diet, sample

Table 3. Antimicrobial resistance test results for 871 fecal Escherichia coli isolates recovered from 74 calves over the 16 -wk sampling period

\begin{tabular}{|c|c|c|c|c|c|c|}
\hline \multirow[b]{2}{*}{ Antimicrobial } & \multicolumn{2}{|c|}{ Susceptible } & \multicolumn{2}{|c|}{ Intermediate } & \multicolumn{2}{|c|}{ Resistant } \\
\hline & Number & $\%$ & Number & $\%$ & Number & $\%$ \\
\hline Ampicillin & 445 & 51.1 & 426 & 48.9 & 0 & 0.0 \\
\hline Ceftiofur & 629 & 72.2 & 7 & 0.8 & 235 & 27.0 \\
\hline Enrofloxacin & 853 & 97.9 & 12 & 1.4 & 6 & 0.7 \\
\hline Florfenicol & 78 & 9.0 & 406 & 46.6 & 387 & 44.4 \\
\hline Gentamicin & 710 & 81.5 & 8 & 0.9 & 153 & 17.6 \\
\hline Oxytetracycline & 180 & 20.7 & 691 & 79.3 & 0 & 0.0 \\
\hline Sulfadimethoxine & 259 & 29.7 & $\mathrm{NI}^{1}$ & NI & 612 & 70.3 \\
\hline Trimethoprim/sulfamethoxazole & 586 & 67.3 & $\mathrm{NI}$ & NI & 285 & 32.7 \\
\hline
\end{tabular}

${ }^{1} \mathrm{NI}=$ not interpreted; Sensititer plate (Thermo Scientific, Waltham, MA) had only 1 dilution, allowing only determination of susceptible or resistant. 
Table 4. Unadjusted mean ( $\pm \mathrm{SD}$ ) antimicrobial resistance scores (ARS) and frequency of multidrug resistance (MDR) for fecal Escherichia coli isolates reported by milk diet and sample event

\begin{tabular}{|c|c|c|c|c|}
\hline \multirow[b]{2}{*}{ Sample event } & \multirow[b]{2}{*}{ Parameter } & \multicolumn{3}{|c|}{ Milk diet ${ }^{1}$} \\
\hline & & $\begin{array}{c}\text { PNM } \\
\text { (5 herds, } 25 \text { calves })\end{array}$ & $\begin{array}{c}\text { MMR } \\
\text { (5 herds, } 25 \text { calves) }\end{array}$ & $\begin{array}{c}\text { NMR } \\
\text { (5 herds, } 24 \text { calves) }\end{array}$ \\
\hline Week 1 & $\begin{array}{l}\text { No. of isolates } \\
\text { ARS }^{2} \\
\text { MDR }^{3}\end{array}$ & $\begin{array}{l}75 \\
2.5 \pm 1.6 \\
39(52.0)\end{array}$ & $\begin{array}{l}75 \\
2.6 \pm 1.6 \\
34(45.3)\end{array}$ & $\begin{array}{l}72 \\
1.3 \pm 1.2 \\
\quad 9(12.5)\end{array}$ \\
\hline Week 3 & $\begin{array}{l}\text { No. of isolates } \\
\text { ARS } \\
\text { MDR }\end{array}$ & $\begin{array}{l}74 \\
3.5 \pm 1.3 \\
63(85.1)\end{array}$ & $\begin{array}{l}75 \\
3.3 \pm 1.3 \\
53(70.7)\end{array}$ & $\begin{array}{l}72 \\
1.1 \pm 1.1 \\
12(16.9)\end{array}$ \\
\hline Week 5 & $\begin{array}{l}\text { No. of isolates } \\
\text { ARS } \\
\text { MDR }\end{array}$ & $\begin{array}{l}75 \\
2.1 \pm 1.7 \\
36(48.0)\end{array}$ & $\begin{array}{c}75 \\
3.0 \pm 1.5 \\
47(62.7)\end{array}$ & $\begin{array}{c}72 \\
1.0 \pm 1.0 \\
6(8.3)\end{array}$ \\
\hline Week 16 & $\begin{array}{l}\text { No. of isolates } \\
\text { ARS } \\
\text { MDR }\end{array}$ & $\begin{array}{c}72 \\
0.7 \pm 0.8 \\
1(1.4)\end{array}$ & $\begin{array}{c}69 \\
1.0 \pm 1.2 \\
13(18.8)\end{array}$ & $\begin{array}{r}66 \\
0.8 \pm 0.9 \\
2(3.0)\end{array}$ \\
\hline
\end{tabular}

${ }^{1} \mathrm{PNM}=$ pasteurized nonsaleable milk; MMR $=$ medicated milk replacer; NMR $=$ nonmedicated milk replacer.

${ }^{2}$ Possible score of 0 to 8 .

${ }^{3}$ Number $(\%)$ of isolates tested that were multidrug resistant (resistant to $\geq 3$ antimicrobials).

time (age), and prior treatment with systemic antimicrobials within $14 \mathrm{~d}$ of sampling - were all associated with the dependent variable ARS $(P<0.05$; Table 6$)$. Housing of preweaned calves, lactating cow herd size, herd average milk yield, and lactating cow housing were not associated with ARS in this final multivariable model, although it should be noted that only one study farm used group housing for preweaned calves. In general, ARS values were lower for fecal E. coli from calves fed NMR, for samples collected at wk 16 (after weaning), and when systemic antimicrobial therapy had not been administered within the 14-d period preceding fecal sample collection. However, because a significant interaction was detected between milk diet and sampling time and between prior systemic antimicrobial treatment and sampling time, the data were subsequently stratified by sampling time and the models reanalyzed to explore the relationship between ARS (dependent variable) and both milk diet and prior systemic antimicrobial treatment at each sample time separately (wk 1, 3, 5, or 16). Within each sampling time, contrast analysis was used to describe differences

Table 5. Results of univariable models investigating factors associated with fecal Escherichia coli antimicrobial resistance score (ARS; possible values 0 to 8 ) and multidrug resistance (MDR; resistant to $\geq 3$ antimicrobials)

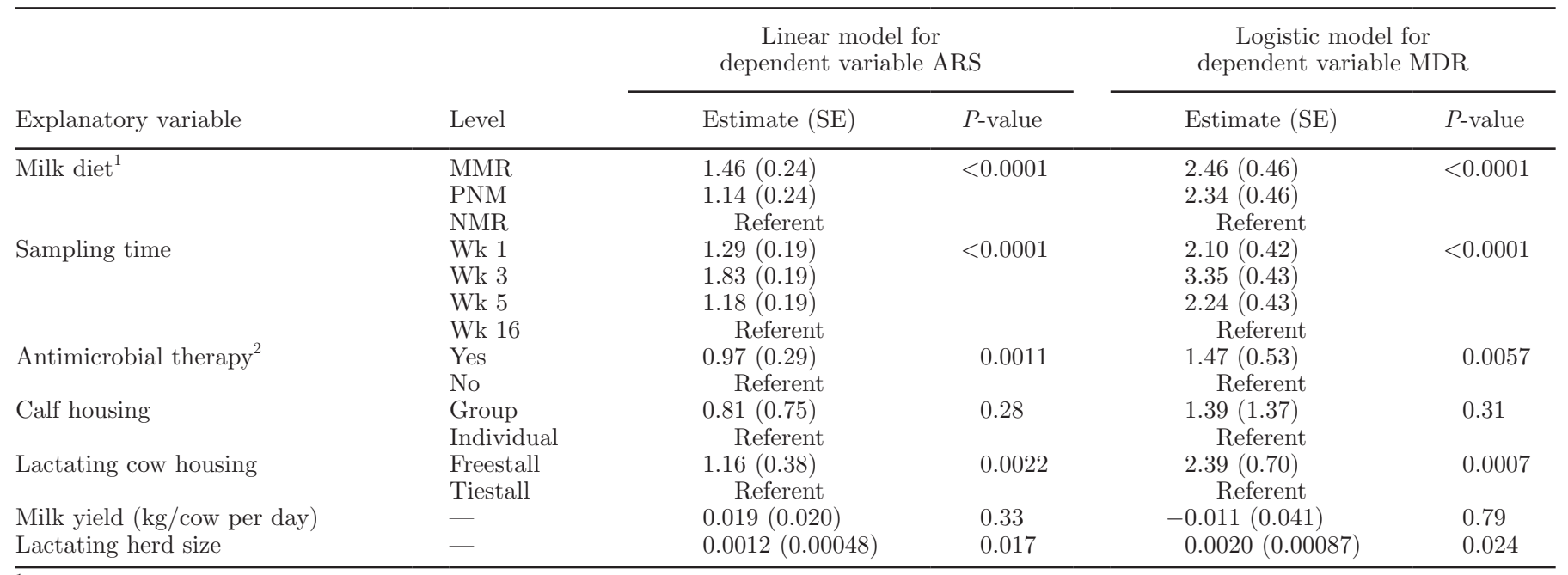

${ }^{1} \mathrm{MMR}=$ medicated milk replacer; $\mathrm{PNM}=$ pasteurized nonsaleable milk; NMR = nonmedicated milk replacer.

${ }^{2}$ Treated with systemic antimicrobial during the 14-d interval preceding the fecal sampling event (yes/no). 
Table 6. Main effects model, before stratified analysis, describing factors associated with fecal Escherichia coli antimicrobial resistance score

\begin{tabular}{|c|c|c|c|}
\hline Explanatory variable & Level & Estimate (SE) & $P$-value \\
\hline \multicolumn{4}{|l|}{ Main effects } \\
\hline Intercept & & $0.80(0.26)$ & 0.0035 \\
\hline \multirow[t]{3}{*}{ Milk diet ${ }^{1}$} & MMR & $0.19(0.37)$ & 0.0002 \\
\hline & PNM & $-0.07(0.36)$ & \\
\hline & NMR & Referent & \\
\hline \multirow[t]{4}{*}{ Sample time } & Wk 1 & $0.49(0.31)$ & $<0.0001$ \\
\hline & Wk 3 & $0.32(0.31)$ & \\
\hline & Wk 5 & $0.10(0.31)$ & \\
\hline & Wk 16 & Referent & \\
\hline \multirow[t]{2}{*}{ Antimicrobial therapy $^{2}$} & Yes & $1.44(0.63)$ & 0.038 \\
\hline & No & Referent & \\
\hline Milk diet $\times$ sample time & Not reported & & $<0.0001$ \\
\hline \multirow[t]{2}{*}{ Antimicrobial therapy $\times$ sample time } & Not reported & & 0.097 \\
\hline & & $\begin{array}{c}\text { Covariate parameter } \\
\text { estimate }\end{array}$ & \\
\hline \multicolumn{4}{|l|}{ Random effects } \\
\hline Herd & & 0.0897 & \\
\hline Calf & & 0.020 & \\
\hline Sample time(calf) & & 0.791 & \\
\hline
\end{tabular}

in the estimates between the 3 milk diets, and adjusted mean (SE) ARS values were generated for each milk diet. The critical $P$-value was adjusted using the Bonferroni correction to account for multiple contrasts among the 3 milk diets (new critical $P$-value $<0.0167$ ). The final results after completing the stratified analysis are presented in Figure 2. For the wk 1, 3, and 5 sampling times, the mean ARS values were greater for fecal E. coli from calves fed either MMR or PNM compared with calves fed NMR, with no difference in ARS values between the MMR and PNM groups at wk 1 or wk 3 (Figure 2). At wk 5 , the mean ARS value (mean $\pm \mathrm{SE}$ ) was greatest for fecal E. coli from calves fed MMR (3.56 $\pm 0.45)$, intermediate for calves fed PNM $(2.64 \pm 0.45)$, and lowest for calves fed NMR $(1.53 \pm 0.45)$. However, by wk 16, the mean ARS values were low for all 3 milk diets, with no difference between MMR $(0.96 \pm 0.32)$, PNM (0.75 \pm 0.32$)$, and NMR (0.84 \pm 0.32 ; Figure 2 ). Treatment with systemic antimicrobials within the 14-d period before sampling was associated with a trend for increased ARS at wk 5 [estimate $(\mathrm{SE})=1.28$ $(0.70) ; P=0.07$, but was not associated with ARS at wk 1,3 , or 16 .

In the final multivariable mixed logistic regression model predicting MDR (yes/no) as the dependent variable, the same 3 explanatory variables of interest, milk diet, sampling time, and systemic antimicrobial treatment within $14 \mathrm{~d}$ preceding sampling, were associated with MDR $(P<0.05$; Table 7$)$. Specifically, the odds for MDR were lower for fecal $E$. coli from calves fed NMR, for samples collected at wk 16 (vs. wk 1,3 , or 5 ), and for calves that were not treated with a systemic antimicrobial in the $14 \mathrm{~d}$ preceding fecal sample collection. However, because of significant interactions between milk diet and sampling time and between previous systemic antimicrobial treatment and sampling time, the data were subsequently stratified by sample time and the models reanalyzed to explore the relationship between odds for MDR in an isolate and both milk diet and previous systemic antimicrobial treatment at each separate sampling time (wk $1,3,5$, or 16). The final results after completing the stratified analysis are presented in Figure 3. For wk 1, 3, and 5, the proportion of $E$. coli isolates with MDR was greatest from calves fed MMR or PNM compared with NMR $(P<$ 0.0167). However, by wk 16, the proportion of $E$. coli isolates with MDR was significantly reduced within the MMR and PNM groups, and there was no difference in the proportion of $E$. coli isolates with MDR from calves fed MMR (7.2\%) compared with PNM (2.8\%) or NMR $(3.9 \%)(P>0.0167$; Figure 3$)$. Treatment with systemic antimicrobials within the 14 -d period before sampling was associated with a trend for increased risk for MDR at wk 3 [estimate $(\mathrm{SE})=1.64$ (0.98); odds ratio $=5.19(95 \% \mathrm{CI}=0.67,35.68) ; P=0.09]$ and with a significant increase in risk for MDR at wk 5 [estimate 


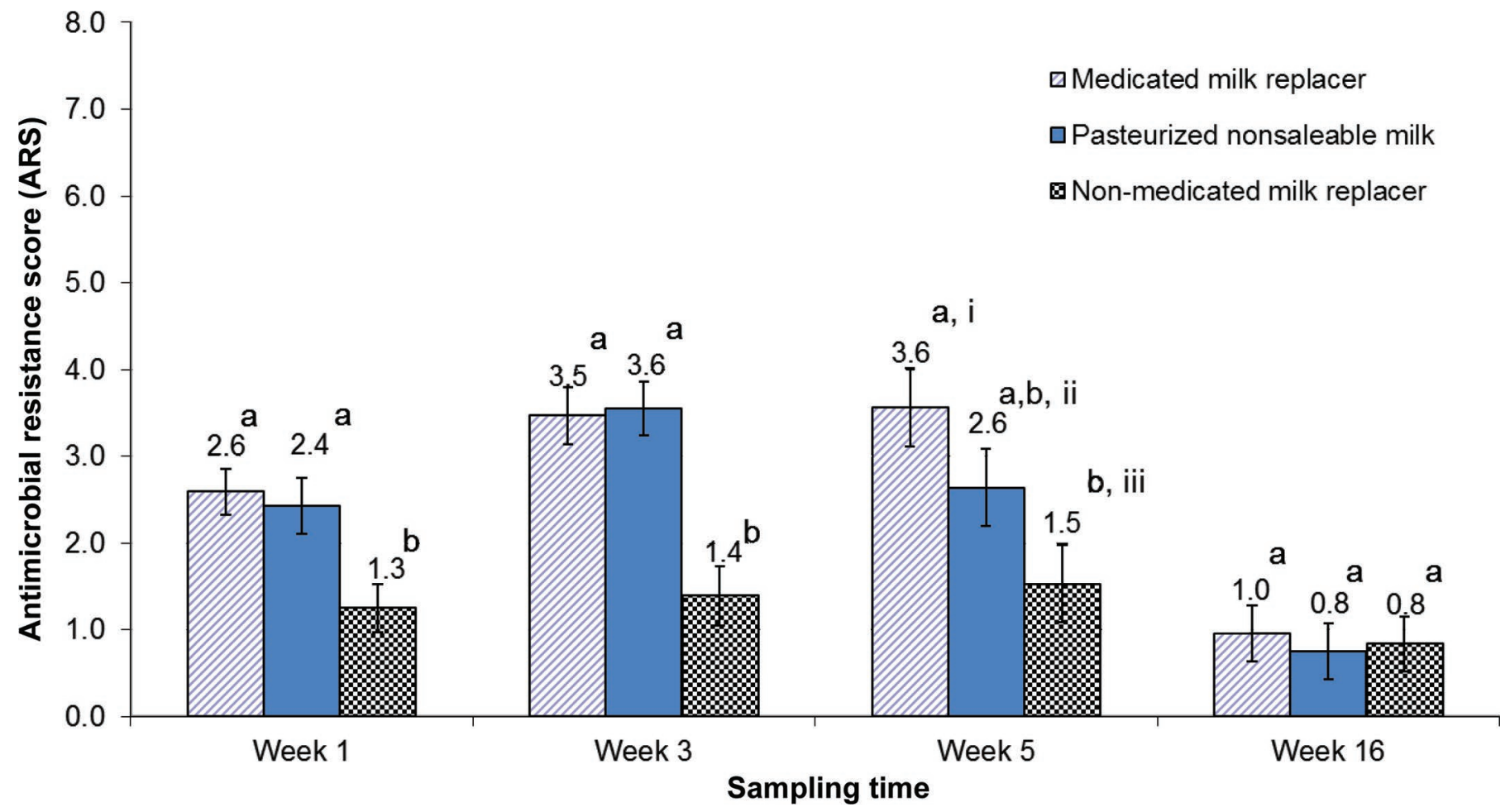

Figure 2. Interaction plot between milk diet and sampling time. Adjusted mean (SE) antimicrobial resistance scores of fecal Escherichia coli from calves fed 3 different milk diets over time. Models adjust for prior treatment with systemic antimicrobial during the 14-d interval preceding the fecal sampling event (yes/no). Different letters $(\mathrm{a}, \mathrm{b})$ indicate a difference between milk diets within sampling time $(P<0.0167$; critical $P$-value adjusted for multiple contrasts); different roman numerals (i, ii, iii) indicate a tendency to differ $(P<0.10)$. Color version available online.

Table 7. Main effects logistic regression model, before stratified analysis, describing factors associated with odds for multidrug resistance in fecal Escherichia coli

\begin{tabular}{|c|c|c|c|}
\hline Explanatory variable & Level & Estimate (SE) & $P$-value \\
\hline \multicolumn{4}{|l|}{ Main effects } \\
\hline Intercept & & $-2.98(0.69)$ & 0.001 \\
\hline \multirow[t]{3}{*}{ Milk diet ${ }^{1}$} & MMR & $1.11(0.89)$ & $<0.0001$ \\
\hline & PNM & $-0.44(1.02)$ & \\
\hline & NMR & Referent & \\
\hline \multirow[t]{4}{*}{ Sample time } & Wk 1 & $0.73(0.84)$ & $<0.0001$ \\
\hline & Wk 3 & $1.00(0.81)$ & \\
\hline & Wk 5 & $-0.40(0.97)$ & \\
\hline & Wk 16 & Referent & \\
\hline \multirow[t]{2}{*}{ Antimicrobial therapy $^{2}$} & Yes & $3.58(1.82)$ & 0.025 \\
\hline & No & Referent & \\
\hline Milk diet $\times$ sample time & Not reported & & 0.012 \\
\hline \multirow[t]{2}{*}{ Antimicrobial therapy $\times$ sample time } & Not reported & & 0.061 \\
\hline & & $\begin{array}{c}\text { Covariance parameter } \\
\text { estimate }(\mathrm{SE})\end{array}$ & \\
\hline \multicolumn{2}{|c|}{ estmate (SE) } & & \\
\hline Herd & & $0.28(0.29)$ & \\
\hline Calf & & $0.40(0.38)$ & \\
\hline Sample time (calf) & & $2.12(0.48)$ & \\
\hline
\end{tabular}

${ }^{1} \mathrm{MMR}=$ medicated milk replacer; $\mathrm{PNM}=$ pasteurized nonsaleable milk; $\mathrm{NMR}=$ nonmedicated milk replacer.

${ }^{2}$ Treated with systemic antimicrobial during the 14-d interval preceding the fecal sampling event (yes/no). 
$(\mathrm{SE})=4.62(2.26) ;$ odds ratio $=101.07(95 \% \mathrm{CI}=$ $1.15,>999.9) ; P=0.043]$, but was not associated with risk for MDR at wk 1 or 16 .

\section{DISCUSSION}

The objective of this prospective cohort study was to describe, under conditions of natural exposure, the relationship between exposure to antimicrobials, both through the milk diet and systemic therapy, and antimicrobial resistance patterns of fecal E. coli in dairy calves pre- and post-weaning. Several previous studies have described associations between exposure to antimicrobials, either through the milk diet or systemic therapy, and antimicrobial resistance patterns in fecal pathogens of preweaned dairy calves (Langford et al., 2003; Berge et al., 2005; Thames et al., 2012; Aust et al., 2013; Pereira et al., 2014a,b; Maynou et al., 2017a,b). Although each of these studies had merit and contributed to the body of knowledge surrounding these questions, they each had limitations. Strengths of our study included the fact that it was conducted in commercial dairy herds under conditions of natural exposure, it investigated associations with exposure to antimicrobials through different milk diets and systemic therapy at the same time, and, most importantly, it was able to follow calves into the postweaning period after exposure to antimicrobials through the milk diets had ended. Additionally, we were able to contrast 3 milk diets that are commonly fed on commercial dairy farms: NMR, MMR, and PNM, whereas many previous studies investigated the effects of only 1 or 2 of these diets. One limitation of the current study is that it focused solely on fecal $E$. coli in pre- and postweaning dairy calves. Additionally, although we tested a panel of 18 antimicrobials, we chose to evaluate only 8 to focus on more clinically relevant antimicrobials. Another limitation to this study is that the sample collection and antimicrobial testing of the nonsaleable milk from the PNM farm was minimal, which hinders further evaluation of the effect on specific antimicrobial resistance. Furthermore, because only one of our 15 herds used group housing before weaning, we were not able to investigate whether calf housing was associated with measures of AMR. However, other studies have identified similar antimicrobial resistance trends in other age classes of ruminants and other types of management systems (Sato et al., 2005; Tragesser et al., 2006; Bosman et al., 2014).

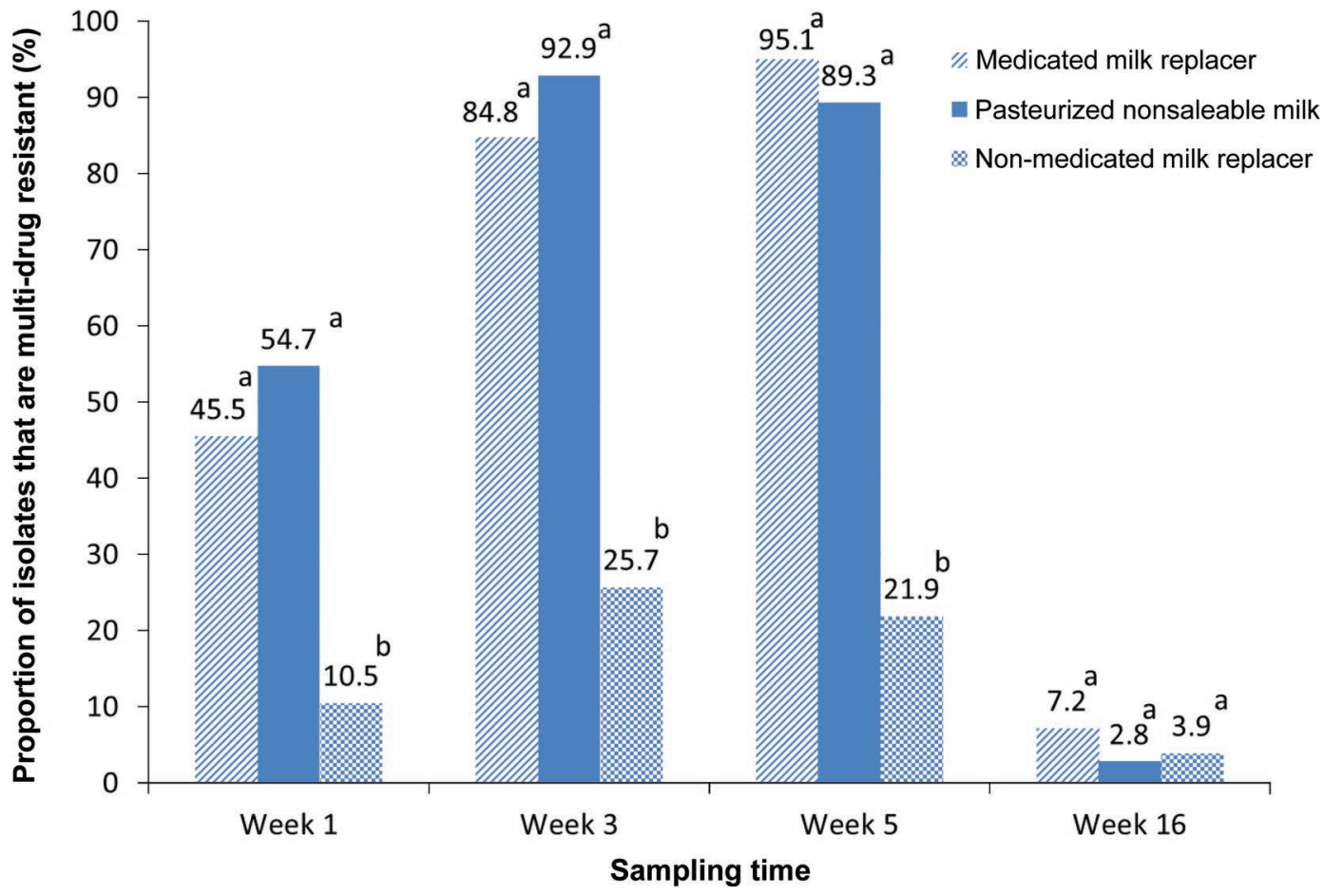

Figure 3. Interaction plot between milk diet and sampling time. Adjusted proportion of fecal Escherichia coli isolates with multidrug resistance from calves fed 3 different milk diets over time. Models adjust for prior treatment with systemic antimicrobial during the 14-d interval preceding the fecal sampling event (yes/no). Different letters $(\mathrm{a}, \mathrm{b})$ indicate a difference between milk diets within sampling time $(P<0.0167$; critical $P$-value adjusted for multiple contrasts). Color version available online. 


\section{Association Between Systemic Antimicrobial Therapy and AMR in Fecal E. coli}

As it relates to the question about the effect of exposure to systemic antimicrobial therapy, our results were generally consistent with previous studies, in that increased AMR was observed in fecal E. coli of calves that were recently treated with systemic antimicrobials (Berge et al., 2005; Pereira et al., 2014a). However, in the current study, increased resistance was observed only if the calf had been treated systemically within the 14-d period preceding fecal sample collection, and it was limited to the wk 5 sampling period, coinciding with the higher risk period for morbidity, and therefore treatment, in dairy calves (USDA, 2010). These findings are consistent with the results of Singer et al. (2008), who demonstrated that increased resistance of fecal E. coli observed during a systemic therapy regimen with ceftiofur in cows was only transient. Berge et al. (2005) also reported a strong association between calf age (at sample event) and ARS, as well as a negative association between the time interval from systemic antimicrobial treatment to sampling and ARS.

\section{Association Between Milk Diet and Age with AMR in Fecal E. coli}

As it relates to the question of the potential effect of exposure to antimicrobials in the milk diet, our results were generally consistent with those of previous studies, in that increased AMR was observed in fecal E. coli of preweaned calves exposed to antimicrobials through the milk diet (Langford et al., 2003; Berge et al., 2005; Aust et al., 2013; Pereira et al., 2014b; Maynou et al., $2017 \mathrm{a}, \mathrm{b})$. Upon stratification of data by sample time, the ARS for fecal $E$. coli for the first 3 sample times (before weaning) were significantly higher for the calves being fed MMR and PNM than for those being fed NMR. Following weaning, however, sample time 4 (wk 16) had the lowest ARS for fecal E. coli of any sampling time point, with no difference in ARS among the 3 different milk diet cohorts. A similar pattern was found for MDR among the 3 milk diets studied.

This transient increase in AMR during the preweaning period, followed by the reemergence of susceptible bacteria postweaning, is most likely due to the discontinuation of exposure to antimicrobials in the MMR and NMR milk diets after weaning. In other words, the selective pressure from exposure to antimicrobials in the milk diet was removed at weaning from the MMR and PNM diets, allowing the susceptible bacteria to flourish in proportions similar to the intestinal environment present in the calves that had previously been fed NMR. These results are consistent with the resistance patterns demonstrated by Maynou et al. (2017a). This same pattern of increased resistance during periods of antimicrobial exposure and decreased resistance after antimicrobial exposure was removed has been shown in other studies (Kaneene et al., 2008; Singer et al., 2008; Maynou et al., 2017b). It is interesting to note that although one herd in our study was feeding chlortetracycline in the calf grower diet fed after weaning, this did not appear to be associated with increased ARS in that herd compared with the other herds.

Despite general consistencies with previous studies, our results differ in some respects from other study findings. For example, when comparing ARS results when feeding PNM and MMR, Berge et al. (2005) reported that fecal E. coli resistance scores were significantly lower for calves fed nonsaleable milk than for those fed the MMR, whereas we found that fecal $E$. coli resistance scores were similar for calves fed MMR and PNM, but were higher for both these groups than for NMR during the preweaning period. Furthermore, in our study, the risk of MDR tended to be higher for calves fed MMR (vs. PNM) at wk 5. Some possible explanations for these discrepancies could include differences in study design, study herd factors, sample sizes, or methods of interpreting resistance data. In particular, one explanation could be differences in the levels of antimicrobials found in the nonsaleable milk of study farms. According to the methods described by Berge et al. (2005), calves in the "nonsaleable milk" cohort of herds were fed hospital milk or fresh milk with no added antimicrobials, although the authors did not report testing the nonsaleable milk to verify the presence or absence of antimicrobials. Conversely, in our study, milk samples collected from 3 of the 5 PNM farms had detectable levels of antimicrobials in all samples because milk from cows treated with antimicrobials was included in the nonsaleable milk pool.

When comparing our r-type data to those of Pereira et al. (2014b), we found a similar distribution of MDR isolates when comparing MMR and PNM to NMR isolates. Pereira et al. (2014b) found that $84 \%$ of the isolates from the drug residue group and only $37 \%$ of the isolates from the "no drug residue" group were MDR, whereas we found that 50 and $47 \%$ of isolates were MDR in the MMR and PNM milk diets, respectively, compared with 10\% MDR isolates in the NMR milk diet group. A very interesting difference between our study and several other studies (Pereira et al., 2011, 2014b; Maynou et al., 2017b) was that we did not find any resistance to ampicillin or oxytetracycline. One possible explanation for differences in findings between studies could be attributed to differences on how the intermediate susceptibility isolates are grouped for analysis. As did Maynou et al. (2017b), we chose a 
conservative approach by grouping the intermediate (I) category with the susceptible (S) isolates, limiting the overinterpretation of resistance. However, Pereira et al. (2014b) chose to combine the intermediate group with the resistant $(\mathrm{R})$ isolates for analysis. Additionally, when classifying isolates as resistant, our group placed isolates in the resistant group only if the MIC broke the resistant cut-off, whereas any MIC results falling between intermediate and resistant were combined into the intermediate group. This conservative approach may have contributed in part to the differences in percentage of resistance in ampicillin and oxytetracycline results among the various studies.

Thames et al. (2012) evaluated the establishment of antimicrobial resistance genes in the feces of calves receiving milk replacer containing no antimicrobials versus subtherapeutic or therapeutic doses of tetracycline and neomycin, with calves being sampled at 6,7 (preweaning), and $12 \mathrm{wk}$ (5 wk after weaning) of age. In that study, although feed antimicrobials had little effect on antimicrobial resistance genes, the authors noted an increase in abundance of several antimicrobial resistance genes in the postweaning (wk 12) sampling event, suggesting that the calf gut environment itself was able to acquire the potential for resistance elements regardless of the antimicrobial content of feed. A plausible explanation for the lack of increased resistance may be that the latter study evaluated only a small number of antimicrobial resistance genes and there may be other resistance genes with significant changes that were not evaluated. In addition, our study focused on phenotypic expression of resistance and not antimicrobial resistance genes. Caution is needed when comparing genotypic resistance (Thames et al., 2012) and phenotypic resistance (our own study), as the first does not necessarily predict the second.

Edrington et al. (2011) reported no change in levels of shedding of E. coli O157:H7 or Salmonella in the feces and no significant change in the antimicrobial susceptibility of these bacteria from preweaning to postweaning when calves were sampled at 2 and 5 d postweaning. In another cross-sectional observational study, Edrington et al. (2012) reported that the prevalence and fecal concentration of MDR E. coli decreased with increasing calf age (by $6 \mathrm{mo}$ ), when subgroups of different calves from 4 farms were sampled at 1,2, 4 , and $8 \mathrm{wk}$ and 4 and 6 mo of age. However, in a separate study of calves from 4 farms, there was no immediate difference in prevalence or fecal concentrations of MDR E. coli when the same calves were sampled $2 \mathrm{~d}$ before and again $2 \mathrm{~d}$ after weaning. Two possible causes for the differences in age-related findings may include the fact that the Edrington et al. (2011, 2012) studies sampled over a very short interval ( 2 or $5 \mathrm{~d}$ ) before collecting the postweaning sample, and the second study reported insignificant amounts of antimicrobials in the nonsaleable milk fed to calves. By comparison, our study allowed for approximately $59 \mathrm{~d}$ between weaning and the 16-wk sampling event. Furthermore, our study found antimicrobials in all samples of PNM tested.

\section{Implications of Study Findings}

Because the association between systemic antimicrobial therapy or exposure to antimicrobials through the milk diet and AMR of fecal E. coli appear to be transient, should these practices be a cause for concern? In one sense, we could argue that the newly resistant bacteria were probably not created by feeding MMR or PNM. Rather, the pressure of feeding antimicrobials in the milk diet or treating calves with systemic antimicrobials may have simply caused a predictable but temporary suppression in the numbers of susceptible $E$. coli shed by the calf. As such, we might argue that we need not be concerned about these practices if susceptibility returns to normal after weaning or after discontinuation of systemic therapy? Although this line of thinking may at first glance appear to be logical, some justifiable concerns remain related to the practice of feeding antimicrobials in either commercial milk replacer or whole milk diets, or treating calves with systemic antimicrobials. One concern is that we do not want farm workers, children, or susceptible individuals potentially put at increased risk of exposure to more-resistant enteric pathogens if exposed to preweaned calves or their environment. A second persistent concern is that continued antimicrobial pressure will increase the possibility of eventual creation of new resistant organisms through a variety of mechanisms including, but not limited to, random mutations or plasmid exchange. This could render certain antimicrobials ineffective in treating human or animal diseases caused by resistant pathogens.

The results of this study may be useful in guiding farm-level practices regarding milk-feeding programs and protocols guiding the prudent use of systemic antimicrobial therapy, as well as to emphasize the importance of sanitation and hygiene protocols for people interacting with calves or their environment. Management strategies - including but not limited to improving colostrum management, providing high planes of milk nutrition, and increased attention to hygiene and sanitation - should be adopted to enhance calf immunity and health, thereby avoiding disease and mitigating the use of antimicrobials to treat systemic disease in calves. Other management strategies (e.g., individual vs. group housing systems for preweaned calves) need to be further evaluated to ascertain their contribution to the creation of disease risk and maintenance 
of antimicrobial resistance, with the goal of optimizing antimicrobial use in dairy operations. These findings, in concert with a 2016 review by the Trust in Animals and Food Safety (TAFS, 2016), provide further rationale for increasing public health and food safety awareness and may be relevant to future FDA policies on the practice of feeding medicated milk replacer or nonsaleable milk on dairy farms. Further studies are required to more closely investigate factors affecting acquisition of resistance to specific drugs or drug classes of importance, such as third-generation cephalosporins (ceftiofur) and fluoroquinolones.

\section{CONCLUSIONS}

Fecal E. coli from calves fed either MMR or PNM had significantly higher ARS and increased odds for detection of MDR or acquisition of antimicrobial resistance elements during the preweaning period (wk 1, 3, 5), compared with calves fed the NMR. However, this effect was transient, with differences between milk diets disappearing after weaning (wk 16), suggesting that once the antimicrobial pressure was removed postweaning, susceptible E. coli bacteria were able to become re-established. Fecal E. coli from calves fed all 3 milk diets had a significantly higher ARS and proportion of isolates with MDR in fecal E. coli in the preweaning versus postweaning period. Systemic antimicrobial therapy within the 14-d period before fecal sampling tended to be associated with increased ARS at wk 5 and with increased odds for MDR at wk 3 and 5 . These findings suggest that exposure to antimicrobials through the milk diet or systemic therapy may result in a transient increase in antimicrobial resistance in fecal $E$. coli, but once the antimicrobial pressure was removed after weaning or discontinuation of systemic therapy, susceptible bacteria were able to flourish again, resulting in an overall decrease in resistance. These results can inform producers regarding the prudent use of antimicrobials and the need to follow established regulatory guidelines with the oversight of a veterinarian.

\section{ACKNOWLEDGMENTS}

This work was supported by a grant from the Minnesota Agricultural Experiment Station. We thank the participating dairy producers for allowing us to collect samples from their farms. We also thank various people at the University of Minnesota who contributed in a variety of ways, including Randall Singer for his consulting expertise, Aaron Rendahl for his statistical consulting expertise, Habner Bastos and Cristiano Gontijo for their technical work, Karen Olsen for her expertise and consulting on the MIC testing, and Jenny Timmer- man along with the Laboratory for Udder Health staff for their technical support on MIC testing. Finally, we thank Mary Bulthaus from DQCI Laboratory (Mounds View, MN) for her consulting expertise and technical work with analysis of the pasteurized nonsaleable milk samples.

\section{REFERENCES}

Aust, V., K. Knappstein, H. J. Kunz, H. Kaspar, J. Wallmann, and M. Kaske. 2013. Feeding untreated and pasteurized waste milk and bulk milk to calves: Effects on calf performance, health status and antimicrobial resistance of faecal bacteria. J. Anim. Physiol. Anim. Nutr. (Berl.) 97:1091-1103.

Berge, A. C. B., E. R. Atwill, and W. M. Sischo. 2005. Animal and farm influences on the dynamics of antimicrobial resistance in faecal Escherichia coli in young dairy calves. Prev. Vet. Med. 69:2538 .

Bosman, A. B., J. A. Wagenaar, J. A. Stegeman, J. C. M. Vernooij, and D. J. Mevius. 2014. Antimicrobial resistance in commensal Escherichia coli in veal calves associated with antimicrobial drug use. Epidemiol. Infect. 142:1893-1904.

CLSI. 2008. Performance Standards for Antimicrobial Disk and Dilution Susceptibility Tests for Bacteria Isolated from Animals; Approved Standard. 3rd ed. CLSI document M31-A3. Clinical and Laboratory Standards Institute, Wayne, PA.

CLSI. 2015. Performance Standards for Antimicrobial Disk and Dilution Susceptibility Tests for Bacteria Isolated From Animals. 3rd ed. CLSI supplement VET01S. Clinical and Laboratory Standards Institute, Wayne, PA.

CLSI. 2018. Performance Standards for Antimicrobial Susceptibility Testing. 28th ed. CLSI supplement M100. Clinical and Laboratory Standards Institute, Wayne, PA.

Edrington, T. S., B. H. Carter, R. L. Farrow, A. Islas, G. R. Hagevoort, T. H. Friend, T. R. Callaway, R. C. Anderson, and D. J. Nisbet. 2011. Influence of weaning on fecal shedding of pathogenic bacteria in dairy calves. Foodborne Pathog. Dis. 8:395-401.

Edrington, T. S., R. L. Farrow, B. H. Carter, A. Islas, G. R. Hagevoort, T. R. Callaway, R. C. Anderson, and D. J. Nisbet. 2012. Age and diet effects on fecal populations and antimicrobial resistance of a multi-drug resistant Escherichia coli in dairy calves. Agric. Food Analyt. Bacteriol. 2:162-174.

FDA (Food and Drug Administration). 2015. Veterinary Feed Directive (VFD). Accessed Jan. 23, 2018. https://www.fda.gov/ AnimalVeterinary/DevelopmentApprovalProcess/ucm071807.htm.

Kaneene, J. B., L. D. Warnick, C. A. Bolin, R. J. Erskine, K. May, and R. Miller. 2008. Changes in tetracycline susceptibility of enteric bacteria following switching to nonmedicated milk replacer for dairy calves. J. Clin. Microbiol. 46:1968-1977.

Langford, F. M., D. M. Weary, and L. Fisher. 2003. Antimicrobial resistance in gut bacteria from dairy calves: A dose response to the level of antimicrobials fed in milk. J. Dairy Sci. 86:3963-3966.

Magiorakos, A.-P., A. Srinivasan, R. B. Carey, Y. Carmeli, M. E. Falagas, C. G. Giske, S. Harbarth, J. F. Hindler, G. Kahlmeter, B. Olsson-Liljequist, D. L. Paterson, L. B. Rice, J. Stelling, M. J. Struelens, A. Vatopoulos, J. T. Weber, and D. L. Monnet. 2012. Multidrug-resistant, extensively drug-resistant and pandrug-resistant bacteria: an international expert proposal for interim standard definitions for acquired resistance. Clin. Microbiol. Infect. $18: 268-281$

Maynou, G., A. Bach, and M. Terré. 2017a. Feeding of waste milk to Holstein calves affects antimicrobial resistance of Escherichia coli and Pasteurella multocida isolated from fecal and nasal swabs. J. Dairy Sci. 100:2682-2694.

Maynou, G., L. Migura-Garcia, H. Chester-Jones, D. Ziegler, A. Bach, and M. Terré. 2017b. Effects of feeding pasteurized nonsaleable milk to dairy calves on phenotypes and genotypes of antimicrobial resistance in fecal Escherichia coli isolates before and after weaning. J. Dairy Sci. 100:7967-7979. 
McEwen, S. A., and P. J. Fedorka-Cray. 2002. Antimicrobial use and resistance in animals. Clin. Infect. Dis. 34(Suppl 3):S93-S106.

Pereira, R. V., J. D. Siler, R. C. Bicalho, and L. D. Warnick. 2014b. In vivo selection of resistant $E$. coli after ingestion of milk with added drug residues. PLoS One 9:e115223. https://doi.org/10.1371/ journal.pone.0115223.

Pereira, R. V., J. D. Siler, J. C. Ng, M. A. Davis, Y. T. Grohn, and L. D. Warnick. 2014a. Effect of on-farm use of antimicrobial drugs on resistance in fecal Escherichia coli of preweaned dairy calves. J. Dairy Sci. 97:7644-7654.

Pereira, R. V. V., T. M. A. Santos, M. L. Bicalho, L. S. Caixeta, V. S. Machado, and R. C. Bicalho. 2011. Antimicrobial resistance and prevalence of virulence factor genes in fecal Escherichia coli of Holstein calves fed milk with and without antimicrobials. J. Dairy Sci. 94:4556-4565.

Sato, K., P. C. Bartlett, and M. A. Saeed. 2005. Antimicrobial susceptibility of Escherichia coli isolates from dairy farms using organic versus conventional production methods. J. Am. Vet. Med. Assoc. 226:589-594.

Singer, R. S., S. K. Patterson, and R. L. Wallace. 2008. Effects of therapeutic ceftiofur administration to dairy cattle on Escherichia coli dynamics in the intestinal tract. Appl. Environ. Microbiol. 74:6956-6962.
TAFS (Trust in Animals and Food Safety). 2016 TAFS Forum. Antimicrobial residues in condemned (waste) milk and its potential impact on public health/food safety. Accessed Dec. 12, 2017. http: //www.tafsforum.org/other-topics.html.

Thames, C. H., A. Pruden, R. E. James, P. P. Ray, and K. F. Knowlton. 2012. Excretion of antimicrobial resistance genes by dairy calves fed milk replacers with varying doses of antimicrobials. Front. Microbiol. 3:139.

Tragesser, L. A., T. E. Wittum, J. A. Funk, P. L. Winokur, and P. J. Rajala-Schultz. 2006. Association between ceftiofur use and isolation of Escherichia coli with reduced susceptibility to ceftriaxone from fecal samples of dairy cows. Am. J. Vet. Res. 67:1696-1700.

USDA. 2010. Dairy 2007: Heifer Calf Health and Management Practices on U.S. Dairy Operations, 2007. \#550.0110. USDA Animal and Plant Health Inspection Service, Veterinary Service, Center for Epidemiology and Animal Health, Fort Collins, CO

USDA. 2014. Dairy 2014: Dairy Cattle Management Practices in the United States, 2014. \#692.0216. USDA Animal and Plant Health Inspection Service, Veterinary Service, Center for Epidemiology and Animal Health, Fort Collins, CO. 